\title{
Water-mediated ionic interactions in protein structures
}

\author{
R Sabarinathan, K Aishwarya, R Sarani, M Kirti Vaishnavi and K Sekar* \\ Bioinformatics Centre, Centre of Excellence in Structural Biology and Bio-computing, Indian Institute of Science, \\ Bangalore 560 012, India
}

*Corresponding author (Fax, +91-080-23600683/23600551; Email, sekar@physics.iisc.ernet.in)

\begin{abstract}
It is well known that water molecules play an indispensable role in the structure and function of biological macromolecules. The water-mediated ionic interactions between the charged residues provide stability and plasticity and in turn address the function of the protein structures. Thus, this study specifically addresses the number of possible water-mediated ionic interactions, their occurrence, distribution and nature found in $90 \%$ non-redundant protein chains. Further, it provides a statistical report of different charged residue pairs that are mediated by surface or buried water molecules to form the interactions. Also, it discusses its contributions in stabilizing various secondary structural elements of the protein. Thus, the present study shows the ubiquitous nature of the interactions that imparts plasticity and flexibility to a protein molecule.
\end{abstract}

[Sabarinathan R, Aishwarya K, Sarani R, Vaishnavi MK and Sekar K 2011 Water-mediated ionic interactions in protein structures. J. Biosci. 36 253-263] DOI 10.1007/s12038-011-9067-4

\section{Introduction}

Water molecules play a vital role in governing biological macromolecules aiding in the stabilization of the threedimensional architecture (Franks 2002; Chaplin 2006; Raschke 2006), dynamics (Otting et al. 1991; Halle 2004; Raschke 2006) and function (Eisenmesser et al. 2005; Smolin et al. 2005; Zhang et al. 2007). Further, the formation of water bridges between the protein complexes enhances water-mediated protein-protein interactions (Langhorst et al. 2000; Davey et al. 2002), protein-DNA interactions (Morton and Ladbury 1996; Grove 2003) and protein-ligand interactions (Poornima and Dean 1995). In addition, these interactions are involved in many biological functions like biomolecular recognition (Papoian et al. 2003), stabilization of interfaces and protein folding and unfolding (Papoian et al. 2004; Levy and Onuchic 2006). In a protein molecule, an ionic interaction is defined as an electrostatic interaction between the polar atoms of the positively (Arg, Lys and His) and negatively (Glu and Asp) charged residues. Ionic interactions play an important role in oligomerization, molecular recognition, domain motion, thermostability and $\alpha$-helix capping (Perutz 1970; Fersht 1972; Barlow and Thornton 1983; Musafia et al. 1995; Xu et al. 1997; Kumar et al. 2000; Kumar and Nussinov 2002; Shankar et al. 2007). Thus, a water-mediated ionic interaction is defined as when one or more water molecules mediate an interaction between a pair of charged residues. For example, disruption of surface salt bridges (a class of ionic interactions) by water molecules in proteins permits protein-DNA interactions (Grove 2003) because it creates the cationic surface complementary to the anionic DNA phosphate in the wrapped complex interface (Saecker and Record 2002).

Hydration of ionic interactions may lower the energy of the molecule (Pratt et al. 1994) as seen in the case of the enzyme cytochrome P450 (Opera et al. 1997). When three-dimensional protein structures were compared both at room and cryo-temperatures, it was found that the room temperature structure contained a bidentate salt bridge, whereas the cryo-temperature structure had a salt bridge mediated by a water molecule (Natesh et al. 2003). This implies that the mediation of water molecule plays a crucial role in the formation of some alternate arrangements in proteins. Recent studies have emphasized on the

Keywords. Plasticity and flexibility; protein complexes; protein-protein interactions; structure packing and stability; water-mediated ionic interactions

Supplementary materials pertaining to this article are available on the Journal of Biosciences Website at http://www.ias.ac.in/jbiosci/ jun2011/Suppl/253supp.pdf 
inclusion of water molecules when modelling protein systems (Papoian et al. 2003; Jiang et al. 2005; Van Dijk and Bonvin 2006) and in hydration-based ligand design (Poornima and Dean 1995). Some studies on the interactions formed by buried (Park and Saven 2005) and surface water molecules have also been attempted (Teyra and Pisabarro 2007; Rodier et al. 2005). These studies take into account not only the water-mediated ionic interactions but also long-range hydrophobic interactions and the interactions between neutral moieties. Although there has been a specific study on the contribution of water-mediated salt bridges that enhances thermal stability of the 'tail' region of GrpE protein from E. coli (Mehl et al. 2007) and thermophilic mutants (Bogin et al. 2002), there is not much information available about the nature and distribution of various types of water-mediated ionic interactions over a large dataset of protein structures available in its archive, Protein Data Bank, or PDB (Berman et al. 2000). Therefore, the present study aims at analysing the frequency of occurrence and distribution of watermediated ionic interactions in secondary structures and the possible role it plays within and between the subunits. Such a study would contribute to the understanding of biomacromolecular interactions as well as reveals some valuable insights into protein stability and flexibility.

\section{Materials and methods}

\subsection{Dataset}

In order to reduce the bias caused by redundancy, a statistical analysis of protein structures required the use of non-redundant data. Thus, in this study, a total of 9577 unique protein chains with low mutual sequence identity of less than $90 \%$ were obtained from the non-redundant data (Hobohm and Sander 1994). While constructing the dataset, only one chain was included from a multimeric protein structure (for example, only one chain was considered in the case of a protein molecule composed of two identical chains in the asymmetric unit with sequence identity of more than $90 \%$ ). Only those X-ray-analysed crystal structures whose resolution was better than or equal to $1.5 \AA$ and $\mathrm{R}$-factor better than or equal to $20 \%$ were selected and included in the dataset. After applying these quality cut-off criteria, the dataset reduced to 1021 chains corresponding to 979 proteins, of which 37 proteins had multiple chains. On the basis of Structural Classification of Proteins (SCOP) classification (Murzin et al. 1995), the protein chains in the dataset represent all protein classes (figure 1). The three-dimensional atomic coordinates of the protein chains were downloaded from the locally available PDB FTP anonymous server, Bioinformatics centre, Indian Institute of Science, India.

\subsection{Methods}

For the purpose of this study, the following simplification was made regarding the water-mediated ionic interactions. Only the crystallographic water molecules present in the PDB files are included for computation. In basic residues, $\mathrm{N}^{\delta 1}$ and $\mathrm{N}^{\varepsilon 2}$ atoms of histidine, $\mathrm{N}^{\zeta}$ atom of lysine and the distal nitrogen atoms $\left(\mathrm{NH} 1, \mathrm{NH} 2\right.$ and $\left.\mathrm{N}^{\varepsilon}\right)$ of arginine were considered as potential hydrogen bond donors. On the other hand $\mathrm{O}^{\delta 1}$ and $\mathrm{O}^{\delta 2}$ of aspartate and $\mathrm{O}^{\varepsilon 1}$ and $\mathrm{O}^{\varepsilon 2}$ of glutamate are considered as potential hydrogen-bond acceptors among the acidic residues. In addition, free nitrogen and carbonyl oxygen atoms present in the $\mathrm{N}$ - and C-terminals of the protein chains were also taken into account for this study. Using hydrogen bond distance ( $2.6 \AA$ to $3.5 \AA$ ) criteria, the charged residues and the water molecules involved in mediating ionic interactions were extracted from the above dataset. Then, the resulting data was validated using the program HBPLUS (McDonald and Thornton 1999) to check for hydrogen bonds between the charged atoms and water molecules by using the two following geometric criteria: (1) the donors and acceptors were seen to exist within the distance of $3.5 \AA$ and the angle between them was greater than $90^{\circ}$ and (2) the solvent accessible area of water molecules involved in the ionic interactions were computed using the program NACCESS (Hubbard and Thornton 1993 ) with a probe radius $1.4 \AA$. Water molecules with accessible surface area less than or equal to $2.5 \AA^{2}$ were considered as internal or buried water molecules, whereas the other water molecules were considered as surface water molecules. The mobility or positional spread of each water molecule was calculated using atomic displacement parameter (often referred to as $\mathrm{B}$-factor). The normalized $\mathrm{B}$-factor was calculated using the formula $\left.\mathrm{B}^{\prime} \mathrm{i}=\left(\mathrm{Bi}^{-}-<\mathrm{B}>\right) / \mathrm{B}\right)$, where $\mathrm{Bi}$ is the $\mathrm{B}$-factor of each atom, $\angle \mathrm{B}>$ is the mean $\mathrm{B}$-factor of protein molecule and $\mathrm{B}$ is the standard deviation of the $\mathrm{B}$ factors (Smith et al. 2003). Water molecules with a low normalized B-factor hold high stability. Secondary structural assignments were predicted using the program STRIDE (Frishman and Argos 1995) and the necessary PERL scripts were developed locally to perform the above calculations.

\section{Results and discussion}

\subsection{Distribution of charged residues}

It is interesting to note that out of 1021 protein chains in the dataset, water-mediated ionic interactions are observed in 821 chains based on the two defined geometric criteria. The average percentage of charged residues (positive and negative) present in the dataset corresponds to $12 \%$ (table 1 ). The number of positively charged residues is approximately equal to the number of negatively charged residues in the 


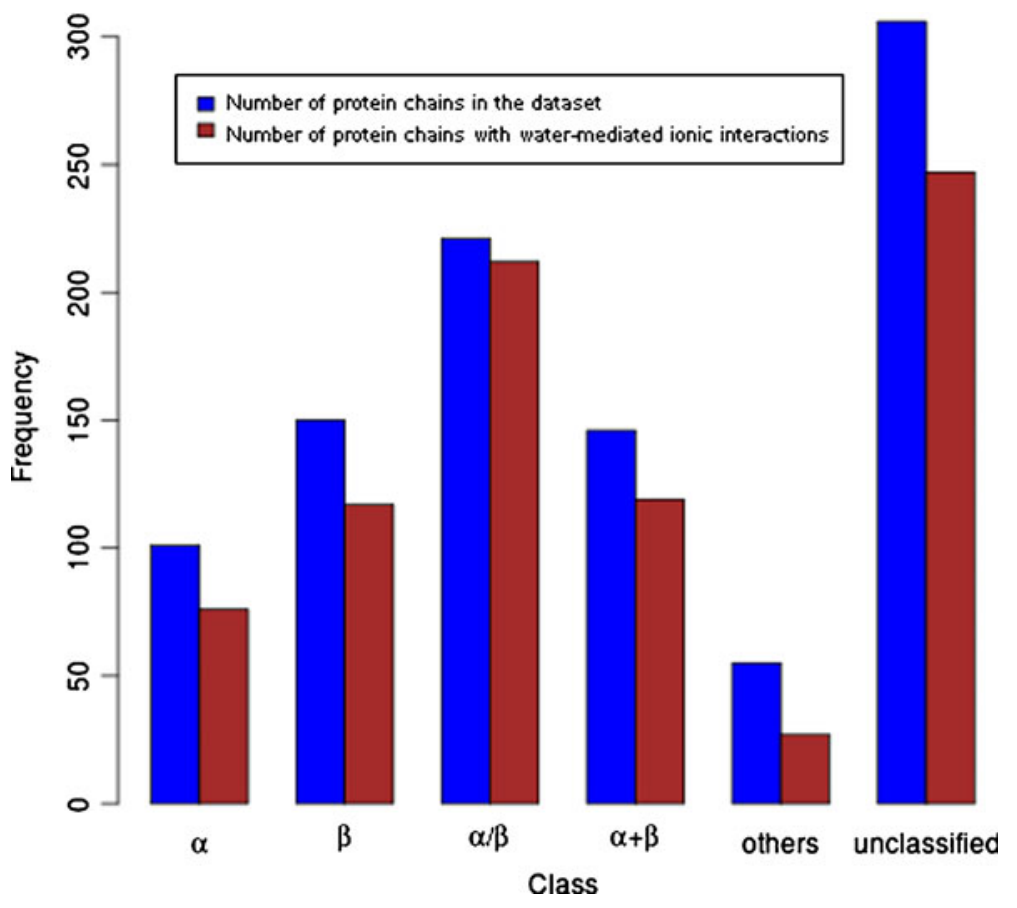

Figure 1. Distribution of different classes (based on SCOP classification) of protein chains used in the present study and the number of protein structures containing water-mediated ionic interactions.

dataset. However, the percentage of positively (32.8\%) charged residues involved in the water-mediated ionic interaction are higher than the participation of negatively (24.6\%) charged residues (table 1). Even though the reason for this difference is solely dependent on the hydrogen bond formation with the mediating water molecule, the positively charged residues have the highest propensity (see following section for details).

\subsection{Distribution of water-mediated ionic interactions}

A total of 3876 water-mediated ionic interactions were observed in 821 protein chains (interactions present within

Table 1. Number of charged residues present in the $90 \%$ nonredundant protein chains and their involvement in the watermediated ionic interaction

\begin{tabular}{lcc}
\hline $\begin{array}{c}\text { Residue } \\
\text { type }\end{array}$ & $\begin{array}{c}\text { Number of charged } \\
\text { residues in the dataset }\end{array}$ & $\begin{array}{c}\text { Number of residues } \\
\text { involved in water-mediated } \\
\text { ionic interactions }\end{array}$ \\
\hline Histidine & $5125(2.37 \%)$ & $371(7.23 \%)$ \\
Arginine & $10434(4.84 \%)$ & $1604(15.37 \%)$ \\
Lysine & $11778(5.46 \%)$ & $1197(10.16 \%)$ \\
Aspartate & $12699(5.89 \%)$ & $1666(13.12 \%)$ \\
Glutamate & $13358(6.19 \%)$ & $1537(11.51 \%)$ \\
\hline
\end{tabular}

and between the subunits). Water-mediated ionic interaction is represented as: $a-\mathrm{HOH}-b$, where ' $a$ ' and ' $b$ ' are the positively and negatively charged residues, respectively, and $\mathrm{HOH}$ is the water molecule that mediates the interaction. The combined pair of residues ('a-b') is termed as a residue pair that contains water-mediated ionic interaction. Out of these, 2020 watermediated ionic interactions were formed between basic residues (His/Lys/Arg) and an acidic aspartate residue. The remaining 1856 interactions were found with glutamate (table 2). Very few water-mediated ionic interactions were observed between the residue pairs His-Asp (248) and HisGlu (190). This might be due to the fact that the imidazole ring of histidine makes it difficult to expose both nitrogen atoms to the solvent at the same time; however, it allows the formation of a single hydrogen bond and is more significant when it is buried. Comparatively more interactions were observed between Lys-Asp and Lys-Glu (table 2). This is because lysine residue has $s p^{3}$-hybridized $\mathrm{N}^{\zeta}$ atom that can form three hydrogen bonds due to its side-chain flexibility. Finally, the highest number of interactions was observed between Arg-Asp and Arg-Glu (table 2). Three nitrogen atoms ( $\mathrm{NH} 1, \mathrm{NH} 2$ and $\mathrm{N}^{\varepsilon}$ ) of argnine contribute to these interactions, in which both $\mathrm{NH} 1$ and $\mathrm{NH} 2$ are often involved in a single or double hydrogen bond formation whereas $\mathrm{N}^{\varepsilon}$ is involved in the formation of a single hydrogen bond. In addition, the hydrogen acceptor present at the other end of aspartate and glutamate residues has two $s p^{2}$-hybridized carboxyl oxygen atoms. Each oxygen atom can accept 
Table 2. Distribution of water-mediated ionic interactions between different charged residues

\begin{tabular}{lcccccc}
\hline & \multicolumn{2}{c}{ Aspartate } & & \multicolumn{2}{c}{ Glutamate } & Total \\
\cline { 2 - 3 } & $\begin{array}{c}\text { Same } \\
\text { subunit }\end{array}$ & $\begin{array}{c}\text { Different } \\
\text { subunits }\end{array}$ & & $\begin{array}{c}\text { Same } \\
\text { subunit }\end{array}$ & $\begin{array}{c}\text { Different } \\
\text { subunits }\end{array}$ & \\
\hline Histidine & 243 & 5 & & 189 & 1 & 438 \\
Arginine & 1088 & 8 & & 953 & 9 & 2058 \\
Lysine & 671 & 5 & & 696 & 8 & 1380 \\
Total & 2020 & & & 1856 & & 3876 \\
\hline
\end{tabular}

hydrogen in the form of two hydrogen bonds. Many residues make at least one hydrogen bond and a significant number of residues make two. Buried residues display an increased propensity towards the formation of two hydrogen bonds with aspartate. This leads to the distribution of different combinations of ionic interactions between charged residues. The water-mediated ionic interactions between the N-terminal nitrogen and C-terminal oxygen atoms were identified in two protein subunits.

The water molecules mediating ionic interactions were identified in both buried and surface regions of the protein structures based on its surface accessible area (Supplementary figure 1). Further, the stability of these water molecules was high, which corresponded to their low normalized B-factor. These interactions are commonly found in all classes of protein structures (figure 1). The distribution of water molecules mediating the ionic interactions in different classes of proteins is shown in figure 2. In all classes of proteins, the number of ionic interactions (figure 2) mediated by the buried and surface water molecules (between the residues pair AspHis and Glu-His) are almost equal. However, the ionic interactions between Asp-Lys, Glu-Lys, Asp-Arg and GluArg are mediated more $(60 \%)$ by surface water molecules than buried water molecules (figure 2). It shows that the distribution of charged residues such as aspartate, glutamate, lysine and arginine on the surface of the protein molecules are highly exposed for water-mediated ionic interactions and leads to the participation in biomolecular interactions.

\subsection{Types of water-mediated ionic interactions}

Water-mediated ionic interactions can be divided into two main types, namely, complete and incomplete, based on the number of atoms involved in the formation ionic interaction (Kumar et al. 2000; Shankar et al. 2007). As mentioned earlier, a water-mediated ionic pair contains a residue pair (an acidic and a basic residue) and a water molecule. In an ionic interaction, either all the side-chain polar atoms from both acidic and basic residues or three atoms (one from basic and two from acidic residues and vice versa) participate in the formation of a water-mediated ionic interaction. In a complete water-mediated ionic interaction, one of the hydrogen bonds formed between water molecule and charged residue is a bifurcated bond. Figure $3 \mathrm{~A}$ depicts the complete water-mediated ionic interaction between the residue pair Arg 173-Asp 48 via HOH 215 in the threedimensional structure of alcohol dehydrogenase [PDB-id: 1O2D] (Schwarzenbacher et al. 2004). When only one atom from the acidic residue and one atom from the basic residue are involved, it is called as an incomplete water-mediated ionic interaction (figure 3B). In the 90\% non-redundant dataset, the percentages of complete and incomplete watermediated ionic interactions are $11.3 \%$ and $88.7 \%$, with respect to the total number of water-mediated ionic interactions observed (supplementary tables 1 and 2). In complete water-mediated ionic interactions, $35 \%$ of the interactions are mediated by the buried water molecules. This helps to stabilize the mobility of water molecules in the core region by forming more than one hydrogen bond with the respective charged residues. In addition, the buried charged atoms get solvated by this interaction and provide

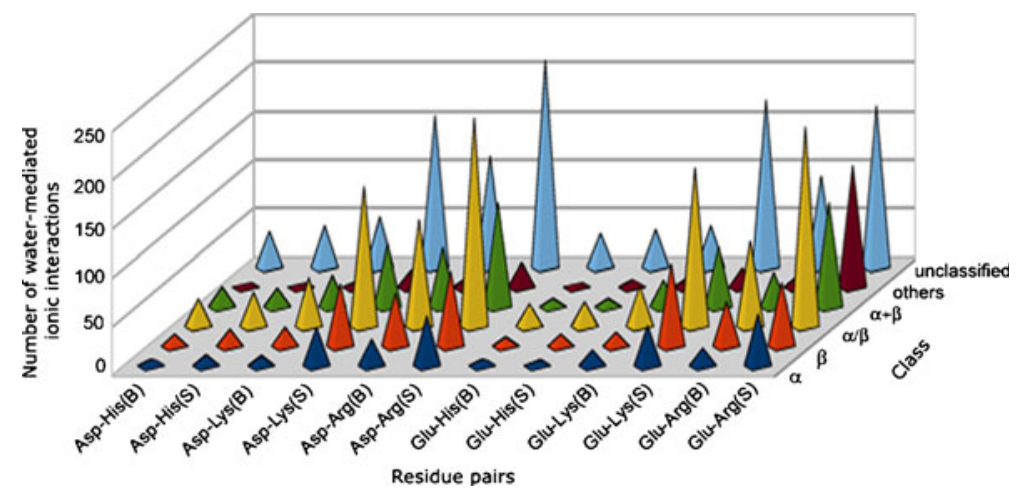

Figure 2. Distribution of the water-mediated ionic interactions mediated by buried (B) and surface (S) water molecules present in different classes of proteins. 

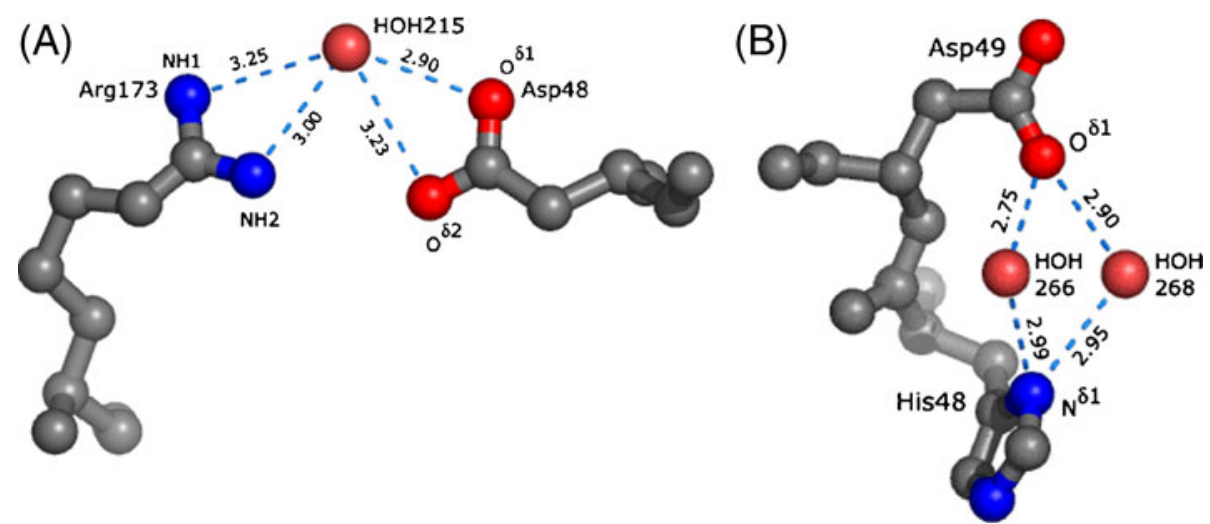

Figure 3. Types of water-mediated ionic interactions: (A) Complete water-mediated ionic interaction observed in the three-dimensional structure of alcohol dehydrogenase [PDB-id: 1O2D], between the residues Arg 173 and Asp 48 (chain A) mediated by the water molecule $\mathrm{HOH}$ 215. (B) Incomplete water-mediated ionic interaction found in the crystal structure of bovine pancreatic phospholipase $\mathrm{A}_{2}$ [PDB-id: 1UNE] is formed between the catalytically important residues (His 48 and Asp 49) via two water molecules, HOH 266 and HOH 268 . The distances are in $\AA$ units.

stability to the inner hydrophobic core of the protein molecule. In incomplete water-mediated ionic interactions, the total number of interactions mediated by surface water molecules is slightly higher when compared with the complete water-mediated ionic interactions. The high distribution of incomplete water-mediated ionic interactions in the surface region helps in biomolecular recognition, which in turn aids in the formation of different protein complexes. It is to be noted that the corresponding percentages of complete and incomplete water-mediated ionic interactions in the $90 \%$ non-redundant dataset is in complete reversal to the direct ionic interaction results published by our group and the values are $58 \%$ and $41 \%$, respectively (Shankar et al. 2007).

Incomplete water-mediated ionic interactions posse significant functionalities as observed in the crystal structure of bovine pancreatic phospholipase $\mathrm{A}_{2}$ (Sekar and Sundaralingam 1999). The ionic interaction is formed between two catalytically important residues His48 and Asp49 (figure 3B). Thus, it can be concluded that, due to the mediation of surface water molecules and the high flexibility, the charged residues on the surface of protein molecules get hydrated. Therefore, the great conformational or steric freedom of surface residues allows the formation of an incomplete water-mediated ionic interaction with great ease.

\subsection{Networks of water-mediated ionic interactions}

Instead of a single residue pair, when three or more charged residues form a water-mediated ionic interaction with at least one residue in common, it is known as a network of water-mediated ionic interactions (figure $4 \mathrm{~A}-$ B). In the present dataset, a total of $20 \%$ of water-mediated ionic interactions were involved in the formation of 148 different types of networks. Some of the network types, which have occurred more than two times, are listed in supplementary table 3. Among the reported 197 networks (supplementary table 3), 34 networks were formed between the polar atoms Asp-Lys-Glu, 31 networks were that of Asp-Arg-Glu combination and the networks between the Asp-Arg-Lys and Glu-Arg-Lys corresponded to 28 and 18 networks, respectively. In some cases, a common residue present in more than one network extends the network. Figure $4 \mathrm{~A}$ depicts the three-dimensional structure of the extended network present within the subunit (chain A) of the nitrobenzene dioxygenase (PDB-id: 2BMO) (Friemann et al. 2005). The interactions are formed between Arg 286Glu 169 via HOH 220, Glu 169- Lys 173 via HOH 219 and Glu 169-His 143 via $\mathrm{HOH} 143$. In the above extended network, Glu 169 is common in all the three incomplete water-mediated ionic interactions and acts as a bridge to link the three individual networks, namely, (a) Arg 286HOH 220-Glu 169-HOH 219-Lys 173, (b) Arg 286-HOH 220-Glu $169-\mathrm{HOH} 143-\mathrm{His} 143$ and (c) Lys $173-\mathrm{HOH}$ 219-Glu 169- HOH 143-His 143 to make an extended network (Pedersen et al. 2004). It has been reported that a total of $10 \%$ of the networks comprised extended networks. The networks within the subunits are mainly helpful in the flexibility of active site and structure stability of the protein molecules. As stated in the literature, the flexibility of the active site in Apo protein tyrosine phosphatase $1 \mathrm{~B}$ is controlled by a network of watermediated interaction (Pedersen et al. 2004). In addition, in aspartic proteinases, the water-molecule-mediated network present in the active site is conserved (Prasad and Suguna 2002). 


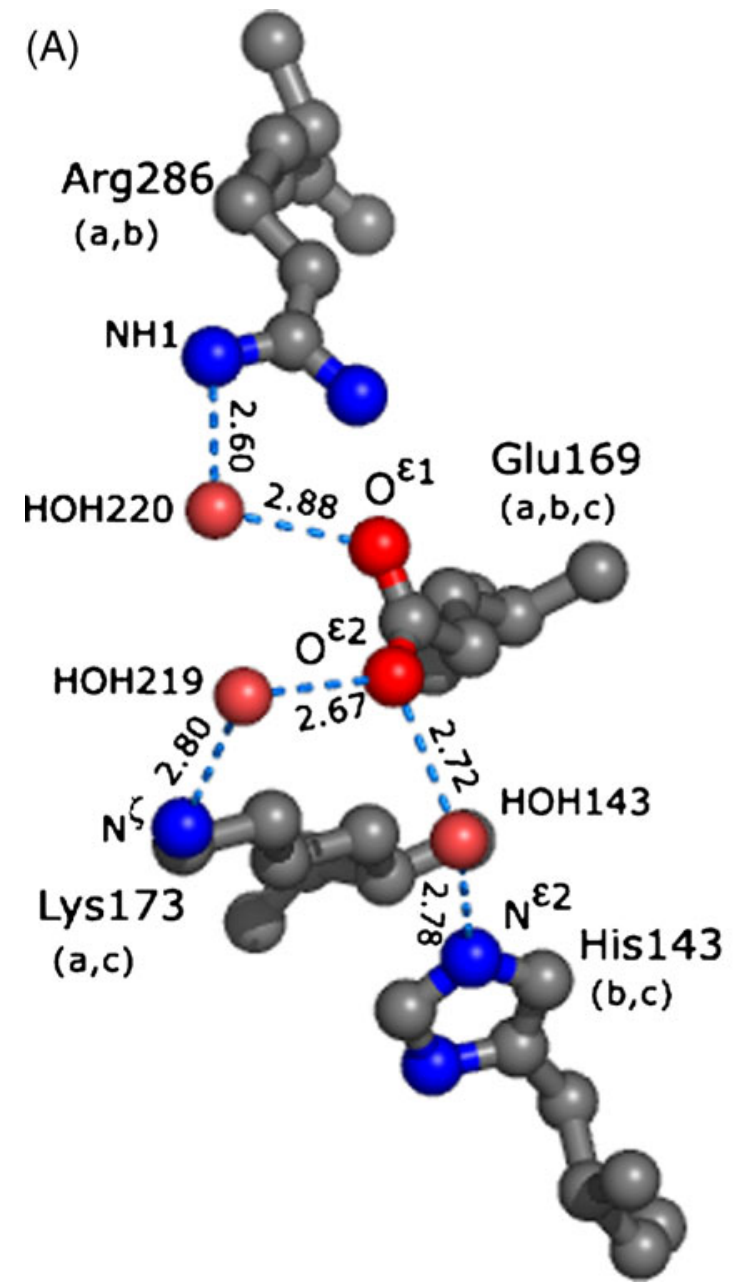

\section{(B)}

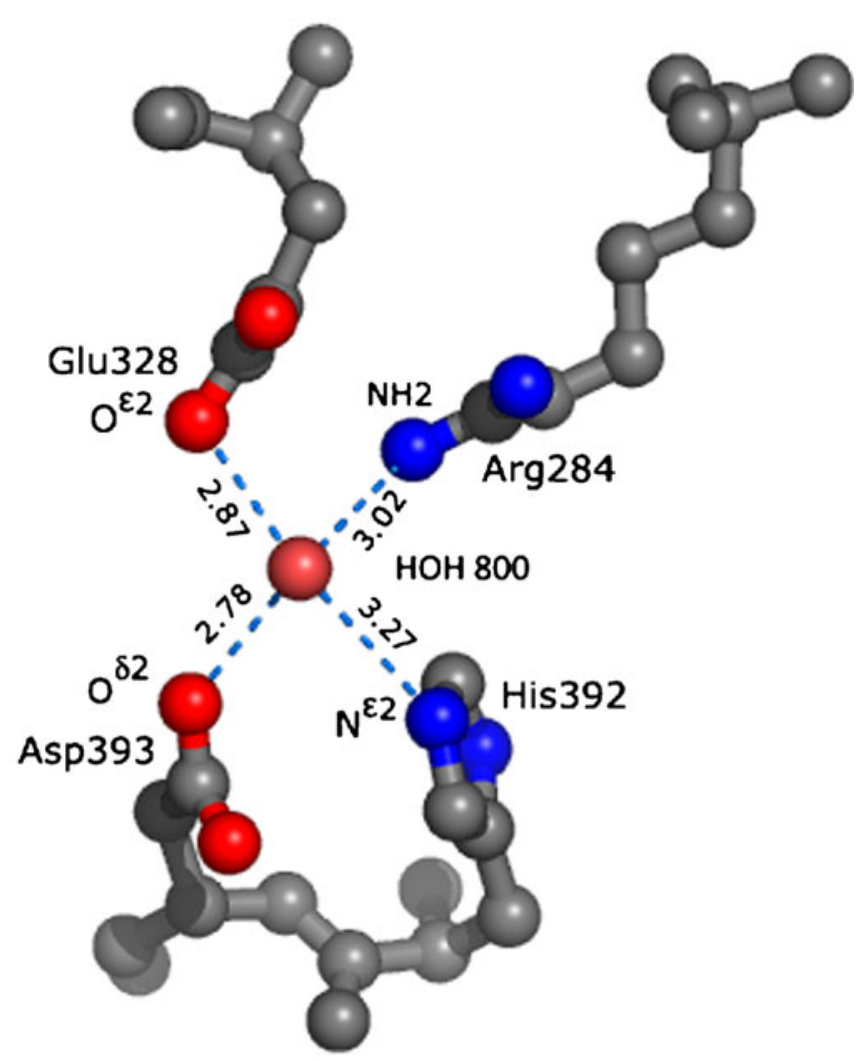

Figure 4. Networks of water-mediated ionic interactions (A) Extended network of water-mediated ionic interactions, in the crystal structure of nitrobenzene dioxygenase (PDB-id: 2BMO). The residue Glu 169 is commonly present among the three different networks (a, b and c). (B) Hubs, a water molecule HOH800 from the crystal structure of amylosucrase [PDB-id: 1G5A] act as a hub for two different water-mediated ionic interactions. The first one is between the Arg 284-Asp 393-Glu 328 via HOH 800 and the second one is between His 392-Asp 393-Glu 328 via the same water molecule HOH 800.

\subsection{Hubs: A special case of networks}

When two or more separated residue pairs appear to share a common (single) water molecule and when each residue pair along with that particular water molecule satisfies the criterion to form a water-mediated ionic interaction, it is called a hub (figure 4B). In the $90 \%$ non-redundant dataset, 20 different water-mediated ionic interactions are involved in the formation of four different hubs within subunits of different protein chains. A typical hub shown in figure 4B is observed in the crystal structure of amylosucrose (PDB-id: 1G5A) (Skov et al. 2001). Here, the water molecule $\mathrm{HOH}$ 800 acts as a hub for the interaction of four charged residues Glu 328, Arg 284, Asp 393 and His 392 in order to form two different water-mediated ionic interactions. Since

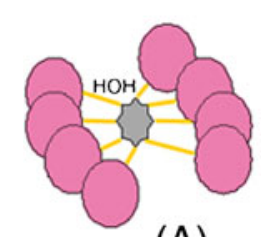

(A)

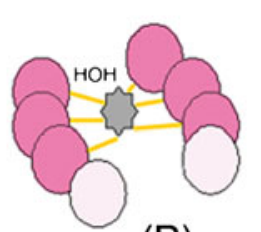

(B)

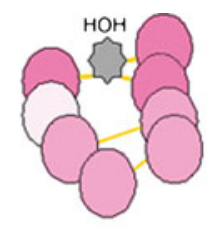

(C)
Figure 5. The diagram shows the possible movements of the residues involved in water-mediated ionic interactions. (A) Hub of water-mediated ionic interactions, (B) some interactions are broken or formed by the entry of water and (C) water molecule escapes and gives rise to ionic interaction formation (last two residue pairs). The dark coloured residues are involved in a water-mediated ionic interaction formation and the lightest ones are not involved in any interaction. 


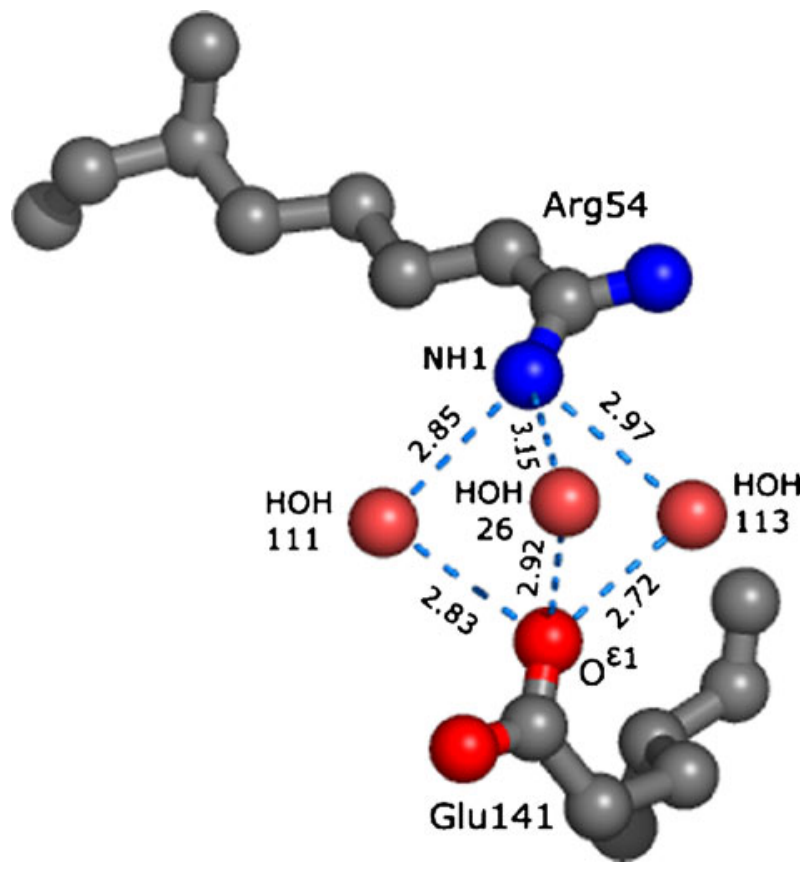

Figure 6. Cluster, a multiple water mediated ionic interaction found between the residues Arg 54 and Glu 141 found by three water molecules ( $\mathrm{HOH} 111, \mathrm{HOH} 26$ and $\mathrm{HOH} 113)$ in the crystal structure of Alpha-tocopherol transfer protein [PDB-id: 1R5L]. The distances are in $\AA$ units.

interfacial water molecules are functionally important (Schimer and Evans 1990), inter-subunit hubs may be located near catalytic sites or may have a specific functionality (like charge redistribution). For example, the transfer of water molecules is involved in protein gating in certain enzymes (Wikstrom et al. 2005). The hubs observed between the dimers of Dps and its related ferritin-like structures helps in the conformational stability of the protein molecule (Ranjani et al. 2008).

As mentioned earlier, the water-mediated interaction between the charged residues increases the plasticity of ionic interactions. The mechanism for hydration in an ionic interaction and its consequent plasticity has been hypothesized in figure 5 . In this example, a hub comprising four residue pairs is depicted in figure 5A. Disruption of ionic interactions upon the entry of a water molecule forms the water-mediated ionic interactions. Similarly, when the water molecule exits, the water-mediated ionic interaction is broken (figure 5B). There is a reformation of the ionic interactions upon complete exit of the water molecule (figure 5C). It clearly shows the flexibility and the plasticity of the water-mediated ionic interactions.

\subsection{Cluster: Multiple water-mediated ionic interactions}

When two or more water molecules mediate an ionic interaction, it is called as multiple water-mediated ionic interaction and the water molecules involved are called cluster of water molecules. The definition of a multiple water-mediated ionic interaction is based on the number of water molecules involved, and so the constituent watermediated ionic interactions can be either complete or incomplete. In the present study, a total of 57 multiple water-mediated ionic interactions were identified comprising 21 residue pairs. Most of the multiple water-mediated ionic interactions were mediated by only two water molecules (figure 3B). The rest of the interactions were mediated by three water molecules. For example, figure 6 depicts a multiple water-mediated ionic interaction between three water molecules ( $\mathrm{HOH} 111, \mathrm{HOH} 26$ and $\mathrm{HOH} 113$ ) and charged residues Arg 54 and Glu 141 in the crystal structure of alpha-tocopherol transfer protein structure (PDB-id: 1R5L) (Min et al. 2003). According to Royer and co-workers, a cluster of interfacial water molecules play a crucial role in the communication between the subunits (Royer et al. 1996). The multiple water-mediated ionic interactions are involved in the catalytic activity of the enzyme bovine pancreatic phospholipase $\mathrm{A}_{2}$ (Shankar and Sekar 2009). Thus, it can be concluded that water-mediated ionic interactions help in maintaining the stability of the structure and facilitates inter-subunit interactions.

Table 3. Distribution of charged residues in various secondary structural elements

\begin{tabular}{|c|c|c|c|c|c|c|}
\hline Secondary structure & Histidine & Arginine & Lysine & Aspartate & Glutamate & Total \\
\hline$\alpha$-helix (H) & 120 & 725 & 481 & 541 & 762 & 2629 \\
\hline 310 -helix (G) & 15 & 70 & 47 & 75 & 77 & 284 \\
\hline$\pi$-helix (I) & 1 & 0 & 0 & 2 & 0 & 3 \\
\hline Extended conformation (E) & 79 & 332 & 271 & 237 & 282 & 1201 \\
\hline Isolated bridge (B) & 2 & 20 & 18 & 17 & 19 & 76 \\
\hline Turn $(\mathrm{T})$ & 71 & 241 & 214 & 499 & 220 & 1245 \\
\hline Coil (C) & 83 & 216 & 166 & 295 & 177 & 937 \\
\hline
\end{tabular}




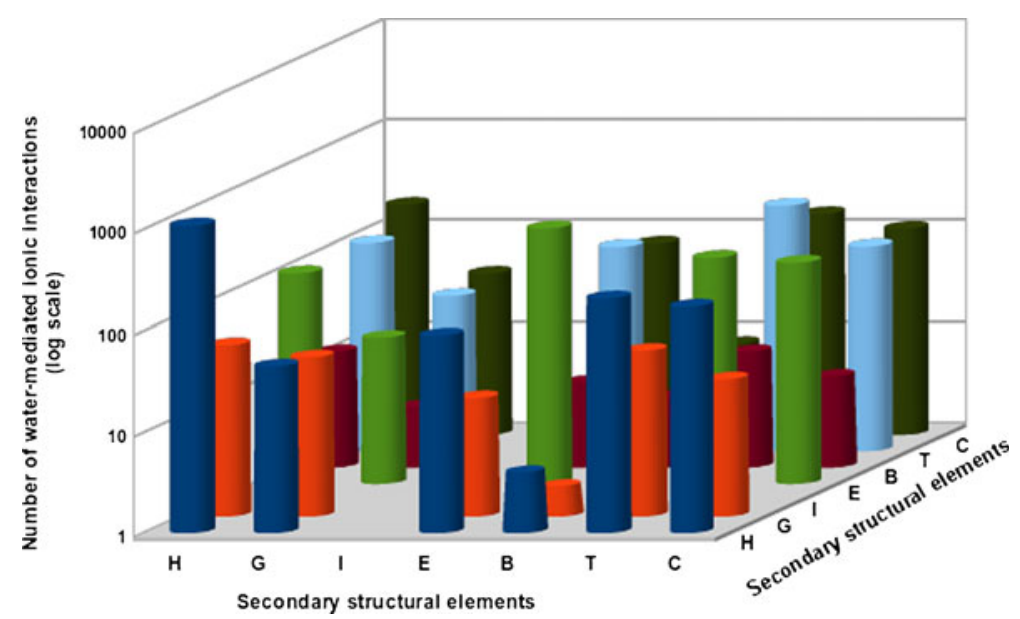

Figure 7. Distribution of water-mediated ionic interactions between the different secondary structural elements. The different secondary structural elements given in the table are H: $\alpha$-helix, G: $3_{10}$-helix, I: $\pi$-helix, E: Extended conformation, B: Isolated bridge, T: Turn and C: Coil.

\subsection{Water-mediated ionic interactions in secondary structures}

In the present study, a total of $5438(85.30 \%)$ charged residues involved in the water-mediated ionic interactions were found in different secondary structures (table 3 ). In addition, the charged residues comprised a total of 3172 $(49.75 \%)$ basic and $3203(50.24 \%)$ acidic residues. The number of residues present in $\alpha$-helices was higher than any other secondary structural elements (table 3). Earlier it had

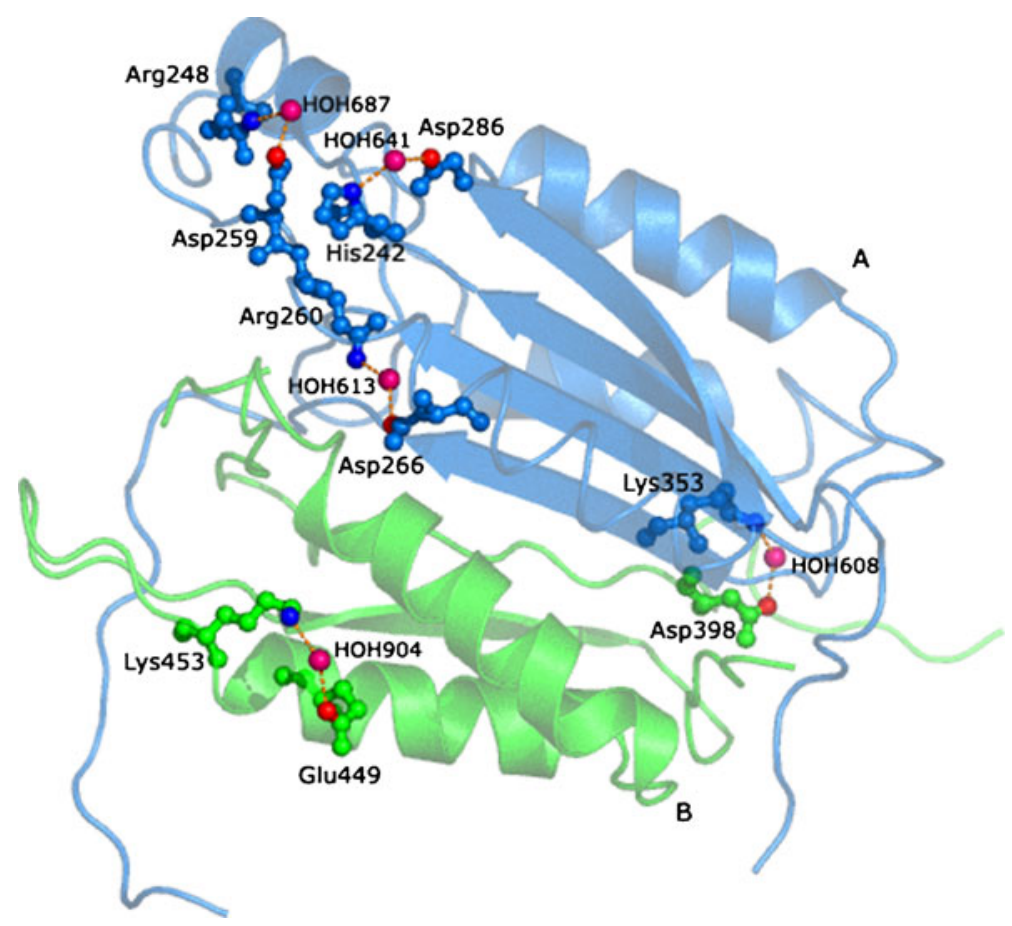

Figure 8. The water-mediated ionic interactions between the residue pairs present in different secondary structural elements of subunits $\mathrm{A}$ and $\mathrm{B}$ in the crystal structure of caspase- 8 protein [PDB-id:1QTN]. 
been reported that direct ion pair interactions are more frequently observed in $\alpha$-helices than in $\beta$-sheets (Shankar et al. 2007). It is a known fact that $\alpha$-helices are the most abundant secondary structural element observed in proteins. Further, water-mediated ionic interactions present between different secondary structural elements were observed within and between the subunits of protein molecules (figure 7). The number of interactions between secondary structural elements like helix-helix and strand-strand corresponded to $31.84 \%$ and $23.91 \%$ (figure 7 ). It is evident that water-mediated ionic interactions are higher in $\alpha$-helices due to intra-helical and inter-helical interactions. Interstrand water-mediated ionic interactions effectively act as inter-sheet interactions. Due to the prevalence of hydrogen bonds, the number of water-mediated ionic interactions required to stabilize a $\beta$-sheet is low. However, $\alpha$-helices are stabilized by electrostatic interaction (Vila et al. 1992). Thus, it can be concluded that these polar intra-helical interactions may well be treated as water-mediated ionic interactions.

The water-mediated ionic interactions observed in other secondary structural elements like helix-coil and strand-coil contributes to $15 \%$ of the total water-mediated ionic interactions. It helps to stabilize the charged residues that are present in an unstable coiled region by introducing a water-mediated ionic interaction between the corresponding residues in the stable secondary structural elements ( $\alpha$-helix or $\beta$-strand). Figure 8 depicts the different secondary structural combinations of water-mediated ionic interactions within and between subunits $\mathrm{A}$ and $\mathrm{B}$ of the protein molecule caspase- 8 protein molecule (PDB-id: 1QTN) (Watt et al. 1999). As shown in the figure, the watermediated ionic interactions among the different secondary structural elements are $\alpha$-helix-coil (A-Arg 248-A-Asp 259), turn- $\alpha$-helix (A-Arg 260-A-Asp 266), coil-coil (AHis 242-A-Asp 286), coil- $\alpha$-helix (B-Lys 453-B-Glu 449) and turn- $\beta$-Strand (A-Lys 353-B-Asp 398). In multisubunit proteins, the percentage of basic and acidic residues (in different secondary structures) forming water-mediated ionic interactions are $51.61 \%$ and $48.39 \%$, respectively (data not shown). Among them, $43 \%$ of the acidic and $50 \%$ of the basic residues are present in $\alpha$-helices, and the corresponding numbers are $10 \%$ and $25 \%$ in $\beta$-strands. A total of 36 different interactions are observed between the subunits. The mediated interaction contains both complete and incomplete water-mediated ionic interactions. The results of different secondary structural combinations show that water-mediated interactions are involved in the anchoring of tertiary structures. These results help to prove the earlier work of Park and Saven (2005). Similarly, the ionic interactions mediated by the water molecules at the interface region also play an important role in the formation of dimers from monomers and other biomolecular complexes.

\section{Conclusion}

In the present study, various patterns of water-mediated ionic interactions formed between the charged residues were classified at the atomic level. The existence of different types of water-mediated ionic interactions, charged networks, hubs and clusters are reported in the present study. Thus, water-mediated ionic interactions have a marked importance in intramolecular interaction, macromolecular association and in biomolecular recognition. Furthermore, the presence of water-mediated ionic interactions in the secondary structural elements confirms their participation in the stability of the same. Water-mediated ionic interactions also play an important role in holding the subunits in multisubunit protein structures. Therefore, the present study is useful to understand and appreciate the role of watermediated ionic interactions in the plasticity and flexibility of protein structures.

\section{Acknowledgements}

The corresponding author (KS) thanks the Department of Biotechnology for financial support. The use of the Bioinformatics Centre and the Interactive Graphics Facility is gratefully acknowledged.

\section{References}

Barlow DJ and Thornton JM 1983 Ion-pairs in proteins. J. Mol. Biol. 168 867-885

Berman HM, Westbrook J, Feng Z, Gilliland G, Bhat TN, Weissig H, Shindyalov IN and Bourne PE 2000 The Protein Data Bank. Nucleic Acids Res. 28 235-242

Bogin O, Levin I, Hacham Y, Tel-Or S, Peretz M, Frolow F and Burstein Y 2002 Structural basis for the enhanced thermal stability of alcohol dehydrogenase mutants from the mesophilic bacterium Clostridium beijerinckii: contribution of salt bridging. Protein Sci. 11 2561-2574

Chaplin M 2006 Do we underestimate the importance of water in cell biology? Nat. Rev. Mol. Cell Biol. 7 861-866

Davey CA, Sargent DF, Luger K, Maeder AW and Richmond TJ 2002 Solvent mediated interactions in the structure of the nucleosome core particle at $1.9 \AA$ resolution. J. Mol. Biol. 319 1097-1113

Eisenmesser EZ, Millet O, Labeikovsky W, Korzhnev DM, Wolf Watz M, Bosco DA, Skalicky JJ, Kay LE and Kern D 2005 Intrinsic dynamics of an enzyme underlies catalysis. Nature (London) 438 36-37

Fersht AR 1972 Conformational equilibria in and chymotrypsin. The energetics and importance of the salt bridge. J. Mol. Biol. 64 497-509

Franks F 2002 Protein stability: the value of 'old literature'. Biophys. Chem. 96 117-127

Friemann R, Ivkovic-Jensen MM, Lessner DJ, Yu CL, Gibson DT, Parales RE, Eklund H and Ramaswamy S 2005 Structural insight into the dioxygenation of nitroarene compounds: the 
crystal structure of nitrobenzene dioxygenase. J. Mol. Biol. 348 1139-1151

Frishman D and Argos P 1995 Knowledge-based protein secondary structure assignment. Proteins 23 566-579

Grove A 2003 Surface salt bridges modulate DNA wrapping by the type II DNA-binding protein TF1. Biochemistry 42 8739-8747

Halle B 2004 Protein hydration dynamics in solution: a critical survey. Philos. Trans. R. Soc. Lond., Ser. B 359 1207-1223

Hobohm U and Sander C 1994 Enlarged representative set of protein structures. Protein Sci. 3 522-524

Hubbard SJ and Thornton JM 1993 NACCESS Computer Program. Department of Biochemistry and Molecular Biology, University College, London.

Jiang L, Kuhlman B, Kortemme T and Baker D 2005 A 'solvated rotamer' approach to modeling water-mediated hydrogen bonds at protein-protein interfaces. Proteins 58 893-904

Kumar S and Nussinov R 2002 Relationship between ion pair geometries and electrostatic strengths in proteins. Biophys. J. 83 1595-1612

Kumar S, Ma B, Tsai CJ and Nussinov R 2000 Electrostatic strengths of salt bridges in thermophilic and mesophilic glutamate dehydrogenase monomers. Proteins 38 368-383

Langhorst U, Backmann J, Loris R and Steyaert J 2000 Analysis of a water mediated protein-protein interactions within RNase T1. Biochemistry 39 6586-6593.

Levy Y and Onuchic JN 2006 Water mediation in protein folding and molecular recognition. Annu. Rev. Biophys. Biomol. Struct. 35 389-415

McDonald IK and Thornton JM 1999 Satisfying hydrogen bonding potential in proteins. J. Mol. Biol. 238 777-793

Mehl AF, Demeler B and Zraikat A 2007 A water mediated electrostatic interaction gives thermal stability to the 'tail' region of the GrpE protein from E. coli. Protein J. 26 239-245

Min KC, Kovall RA and Hendrickson WA 2003 Crystal structure of human alpha-tocopherol transfer protein bound to its ligand: implications for ataxia with vitamin E deficiency. Proc. Natl. Acad. Sci. USA 100 14713-14718

Morton CJ and Ladbury JE 1996 Water-mediated protein-DNA interactions: the relationship of thermodynamics to structural detail. Protein Sci. 5 2115-2118

Murzin AG, Brenner SE, Hubbard T and Chothia C 1995 SCOP: a structural classification of proteins database for the investigation of sequences and structures. J. Mol. Biol. 247 536-540

Musafia B, Buchner V and Arad D 1995 Complex salt bridges in proteins: statistical analysis of structure and function. J. Mol. Biol. 254 761-770

Natesh R, Manikandan K, Bhanumoorthy P, Viswamitra MA and Ramakumar S 2003 Thermostable xylanase from Thermoascus aurantiacus at ultrahigh resolution $(0.89 \mathrm{~A})$ at $100 \mathrm{~K}$ and atomic resolution $(1.11 \mathrm{~A})$ at $293 \mathrm{~K}$ refined anisotropically to smallmolecule accuracy. Acta Cryst. D59 105-117

Opera TI, Hummer G and Garcia AR 1997 Identification of a functional water channel in cytochrome P450 enzymes. Proc. Natl. Acad. Sci. USA 94 2133-2138

Otting G, Liepinsh E and Wuthrich K 1991 Protein hydration in aqueous solution. Science $254974-980$

Papoian GA, Ulander J and Wolynes PG 2003 Role of water mediated interactions in protein-protein recognition landscapes. J. Am. Chem. Soc. 125 9170-9178
Papoian GA, Ulander J, Eastwood MP, Luthey-Schulten Z and Wolynes PG 2004 Water in protein structure prediction. Proc. Natl. Acad. Sci. USA $1013352-3357$

Park S and Saven JG 2005 Statistical and molecular dynamics studies of buried waters in globular proteins. Proteins $60450-463$

Pedersen AK, Peters GH, MØller KB, Lversen LF and Kastrup JS 2004 Water-molecule network and active-site flexibility of apo protein tyrosine phosphatase 1B. Acta Cryst. D60 1527-1534

Perutz MF 1970 Stereochemistry of cooperative effects in haemoglobin. Nature (London) 228 726-739

Poornima CS and Dean PM 1995 Hydration in drug design. 1. Multiple hydrogen-bonding features of water molecules in mediating protein-ligand interactions. J. Comput. Aided. Mol. Des. 9 500-512

Prasad BV and Suguna K 2002 Role of water molecules in the structure and function of aspartic proteinases. Acta Cryst. D58 250-259

Pratt LR, Hummer G and Garcia AR 1994 Ion pair potentials-ofmean-force in water. Biophys. Chem. 51 147-165

Ranjani CV, Rangarajan S, Michael D, Roy S and Sekar K 2008 Role of water molucules and ion pairs in Dps and related ferritin-like structures. Int. J. Biol. Macromol. 43 333-338

Raschke TM 2006 Water structure and interactions with protein surfaces. Curr. Opin. Struct. Biol. 16 152-159

Rodier F, Bahadur RP, Chakrabarti P and Janin J 2005 Hydration of protein-protein interfaces. Proteins $6036-45$

Royer WE, Pardanani A, Gibson QH, Peterson ES and Friedman JM 1996 Ordered water molecules as key allosteric mediators in a cooperative dimeric hemoglobin. Proc. Natl. Acad. Sci. USA 93 14526-14531

Saecker RM and Record MR Jr 2002 Protein surface salt bridges and paths for DNA wrapping. Curr. Opin. Struct. Biol. 12 311-319

Schimer T and Evans PR 1990 Structural basis of the allosteric behaviour of phosphofructokinase. Nature (London) 343 140-145

Schwarzenbacher R, Von Delft F, Canaves JM, Brinen LS, Dai X, Deacon AM, Elsliger MA, Eshaghi S, et al 2004 Crystal structure of an iron-containing 1,3-propanediol dehydrogenase (TM0920) from Thermotoga maritima at $1.3 \mathrm{~A}$ resolution. Proteins 54 174-177

Sekar K, and Sundaralingam M 1999 High-resolution refinement of the orthorhombic bovine pancreatic phospholipase $\mathrm{A}_{2} /$ span. Acta Cryst. D55 46-50

Shankar BA G, Sarani R, Michael D, Mridula P, Ranjani CV, Sowmiya G, Vansundhar B, Sudha P, Jeyakanthan J, Velmurugan D and Sekar K 2007 Ion pairs in non-redundant protein structures. J. Biosci. 32 692-704

Shankar PK and Sekar K 2009 Structural and functional role of water molecules in bovine pancreatic phospholipase $\mathrm{A}_{2}$ : A datamining approach. Acta Cryst. D65 74-84

Skov LK, Mirza O, Henriksen A, De Montalk GP, RemaudSimeon M, Sarcabal P, Willemot RM, Monsan P and Gajhede M 2001 Amylosucrase, a glucan-synthesizing enzyme from the alpha-amylase family. J. Biol.Chem. 276 25273-25278

Smith DK, Radivojac P, Obradovic Z, Dunker AK, Zhu G 2003 Improved amino acid flexibility parameters. Protein Sci. 12(5) 1060-1072

Smolin N, Oleinikova A, Brovchenko I, Geiger A and Winter R 2005 Properties of spanning water networks at protein surfaces. J. Phys. Chem. B 109 10995-11005 
Teyra J and Pisabarro MT 2007 Characterization of interfacial solvent in protein complexes and contribution of wet spots to the interface description. Proteins 67 1087-1095

Van Dijk ADJ and Bonvin AMJJ 2006 Solvated docking: introducing water into the modelling of biomolecular complexes. Bioinformatics 22 2340-2347

Vila J, Williams RL, Grant JA, WÓjcik J and Scheraga HA 1992 The intrinsic helix-forming tendency of L-alanine. Proc. Natl. Acad. Sci. USA 89 7821-7825

Watt W, Koeplinger KA, Mildner AM, Heinrikson RL, Tomasselli AG and Watenpaugh KD 1999 The atomic-resolution structure of human caspase-8, a key activator of apoptosis. Structure 71135 1143

Wikstrom M, Ribacka C, Molin M, Laakkonen L, Verkhovsky M and Puustinen A 2005 Gating of proton and water transfer in the respiratory enzyme cytochrome c oxidase. Proc. Natl. Acad. Sci. USA 102 10478-10481

Xu D, Tsai CJ and Nussinov R 1997 Hydrogen bonds and salt bridges across protein-protein interfaces. Protein Eng. 10 999-1012

Zhang L, Wang L, Kao YT, Qiu W, Yang Y, Okobiah O and Zhong D 2007 Mapping hydration dynamics around a protein surface. Proc. Natl. Acad. Sci. USA 104 18461-18466

MS received 31 January 2011; accepted 11 April 2011

ePublication: 16 May 2011

Corresponding editor: Амit ChATTOPADHYAY 